\title{
Estimulação elétrica neuromuscular em cães submetidos à imobilização rígida temporária da articulação fêmoro-tíbio-patelar
}

\author{
Neuromuscular electrical stimulation in dogs submitted to rigid temporary immobilization of \\ femmoral-tibial-patellar joint
}

\author{
Soraia Figueiredo de Souza ${ }^{\mathrm{I}}$ Alexandre MazzantiII Alceu Gaspar Raiser ${ }^{\mathrm{II}}$ Fabiano Zanini Salbego ${ }^{\mathrm{I}}$ \\ Charles Pelizzari I Danieli Brollo Martins ${ }^{I}$ Raquel Rubia Rech' Sonia Terezinha dos Anjos Lopes ${ }^{I I}$ \\ Diego Vilibaldo Beckmann" Lucilene Bernardi de Souza ${ }^{\text {III }}$ Marina Gabriela Carvalho Mori da Cunha ${ }^{\text {III }}$ \\ Rafael Festugatto $^{\text {III }}$ Rosmarini Passos dos Santos ${ }^{\text {III }}$ Ana Paula da Silva ${ }^{\text {III }}$
}

RESUMO

Com o objetivo de avaliar o efeito da estimulação elétrica neuromuscular (EENM) de baixa freqüência sobre o músculo vasto lateral, foram utilizados 11 cães, agrupados aleatoriamente em três grupos, denominados de I ou controle, de II (EENM após imobilização) e de III (EENM durante e após imobilização). A articulação fêmoro-tíbio-patelar direita foi imobilizada por 30 dias pelo método de transfixação percutânea tipo II. Os cães do grupo III iniciaram as sessões de eletroterapia, três vezes por semana, durante (30 dias) e após a imobilização (60 dias); e os cães dos grupos II após a remoção da imobilização rígida temporária. Foram avaliadas a mensuração da circunferência da coxa, a goniometria do joelho, os graus de claudicação, as enzimas creatina-quinase (CK) e a aspartato-amino-transferase (AST) e a morfometria das fibras musculares longitudinais do vasto lateral. A análise clínica dos graus de claudicação foi realizada diariamente. A medida da circunferência de coxa, a goniometria e a biópsia do músculo vasto lateral foram realizadas nos tempos zero, 30, 60 e 90 dias após imobilização. As amostras de sangue para avaliação da CK e da AST foram coletadas antes, imediatamente depois, em 6, 24 e 48 horas após a EENM, nos dias zero, 1, 7, 30, 45, 60, 75 e 90. A EENM foi empregada no músculo quadríceps femoral numa freqüência de $50 \mathrm{~Hz}$, com duração de pulso de 300 milisegundos e relação "on time/off time” de 1:2. Não houve diferença significativa nos graus de claudicação, nos valores de circunferência da coxa, na goniometria e no comportamento das enzimas CK e AST entre os grupos. Foi observada maior hipertrofia das fibras musculares longitudinais nos cães do grupo II $(P=0,0005)$, seguidos pelos cães do grupo III. Pode-se concluir que a EENM de baixa freqüência ocasiona hipertrofia do músculo vasto lateral em cães após a imobilização rígida temporária da articulação do joelho, recomendando-se o seu uso em animais com atrofia muscular.
Palavras-chave: cão, imobilização, hipertrofia, músculo, reabilitação.

\section{ABSTRACT}

This study was aimed at evaluating the effects of neuromuscular electrical stimulation (NMES) of low frequency on the vastus lateralis muscle. Eleven dogs were randomly placed in 3 groups: I (control), II (NMES post immobilization), and III (NMES during and post immobilization). The right femoral-tibial-patellar joint was immobilized for 30 days by the percutaneous transfixation type II method. The dogs from group III were placed on electrotherapy 3 times a week during (30 days) and post-immobilization (60 days) and the dogs from group II post-removal of the temporary rigid immobilization. The parameters evaluated consisted of: measurement of thigh circumference, joint motion using a goniometer, gait analysis, creatine kinase (CK) and aspartate aminotransferase (AST) levels and morphometry of the longitudinal muscle fibers of vastus lateralis muscle. The gait analysis was performed daily and the circumference of the thigh, joint motion, and muscle biopsy were performed on days 0, 30, 60 and 90 post-immobilization. The blood samples for CK and AST were collected before and immediately after 6, 24 and 48 hours post-NMES on days 0, 1, 7, 30, 45, 60, 75 and 90. The NMES was employed on the femoral quadriceps muscle group with a frequency of $50 \mathrm{~Hz}$ post-duration of $300 \mathrm{msec}$ on an on-time/off-time ratio of 1:2. There was no significant difference on the gait analysis, thigh circumference, joint motion, and CK and AST values. The greatest hypertrophy change was observed on longitudinal fibers of the dogs from group II $(P=0.0005)$, followed by the dogs from group III. NMES of low frequency causes hypertrophy of the vastus lateralis muscle in dogs post-temporary immobilization of the stifle joint, recommending its use in animals with muscular atrophy.

IPrograma de Pós-graduação em Medicina Veterinária, Centro de Ciências Rurais (CCR), Universidade Federal de Santa Maria (UFSM), 97105-900, Santa Maria, RS, Brasil. E-mail: alexamazza@yahoo.com. Autor para correspondência.

IIDepartamento de Clínica de Pequenos Animais, CCR, UFSM, Santa Maria, RS, Brasil.

IIICurso de Medicina Veterinária. CCR, UFSM, Santa Maria, RS, Brasil. 
Key words: dogs, immobilization, hypertrophy, muscle, rehabilitation.

\section{INTRODUÇÃO}

A fixação esquelética externa empregada para imobilizar uma articulação serve para vários propósitos (ANDERSON \& CONSTANTINESCU, 1998), como para reparo de tendão (RAISER, 2000), de patela (MAZZANTI et al., 2004), de ligamentos e para a redução de fratura distal de membros (ANDERSON \& CONSTANTINESCU, 1998).

A Estimulação Elétrica Neuromuscular (EENM) é a ação de estímulos elétricos terapêuticos aplicados sobre o tecido muscular, através do sistema nervoso periférico íntegro (BRASILEIRO et al., 2002). Ela é empregada comumente na terapia física para promover aumento da taxa de movimentação e de força muscular, reeducação muscular, correção de anormalidades estruturais, melhora no tônus muscular, aumento da função, controle da dor, aceleração do processo de cicatrização, redução de edema e espasmo muscular e administração transdérmica de medicamentos (JOHNSON \& LEVINE, 2004).

Entre as seqüelas encontradas após a imobilização rígida temporária de uma articulação, podese citar a atrofia muscular (MORRISSEY et al., 1985; CARVALHO, 2001; MAZZANTI et al., 2004). Este efeito pode ser amenizado se forem empregadas modalidades terapêuticas como a EENM, com o intuito de evitar atrofia ou de promover hipertrofia muscular. Uma dúvida, no entanto, quanto a este procedimento reside no momento ideal da aplicação, ou seja, durante ou após a remoção da imobilização articular.

Diante disso, o objetivo deste estudo é avaliar o efeito da imobilização rígida temporária da articulação do joelho, por um período de 30 dias, sobre os músculos da coxa em cães, e se a EENM, de baixa freqüência evita a atrofia muscular ou promove ganho de massa muscular em cães durante, durante e após e após a imobilização rígida temporária da articulação do joelho.

\section{MATERIAL E MÉTODOS}

Foram utilizados 12 cães, sem raça definida, independentemente do sexo, pesando entre 12 e $25 \mathrm{~kg}$, obtidos no Biotério Central da Universidade Federal de Santa Maria. Foram vermifugados com pamoato de pirantel/praziquantel ${ }^{\mathrm{a}}$ na dose de $25 \mathrm{mg} \mathrm{kg}^{-1}$ de peso corporal, dose esta repetida após 15 dias. Após isso, foram anestesiados com tiopental sódico ${ }^{\mathrm{b}}\left(12,5 \mathrm{mg} \mathrm{kg}^{-1}\right)$ e avaliados por estudo radiográfico simples da articulação coxofemoral e fêmoro-tíbio-patelar, em incidência ventro-dorsal.

Os cães foram distribuídos ao acaso em três grupos de igual número, denominados de Grupo I ou Controle; Grupo II ou EENM após imobilização, no qual os cães foram submetidos à EENM uma vez ao dia, com intervalo mínimo de 24 horas entre as sessões, três vezes por semana, até os 60 dias após imobilização, com início das sessões 48 horas após a remoção do fixador; e o Grupo III ou EENM durante e após imobilização para aqueles que foram submetidos à EENM uma vez ao dia, três vezes por semana, por um período de 90 dias, ou seja, durante (30 dias) e após a retirada da imobilização (60 dias).

Cada animal foi submetido à tricotomia da coxa e pré-medicado com sulfato de morfina ${ }^{c}(0,5 \mathrm{mg}$ $\left.\mathrm{kg}^{-1}\right)$ associado a maleato de acepromazina ${ }^{\mathrm{d}}(0,05 \mathrm{mg}$ $\left.\mathrm{kg}^{-1}\right)$. A anestesia foi induzida com propofole $\left(5,0 \mathrm{mg} \cdot \mathrm{kg}^{-1}\right)$ e mantida com halotano ${ }^{\mathrm{f}}$. A analgesia transoperatória foi assegurada com cloridrato de fentanila ${ }^{\mathrm{g}}(0,002 \mathrm{mg}$ $\left.\mathrm{kg}^{-1}\right)$. A anti-sepsia do campo operatório foi realizada com álcool-iodo-álcool e administrada com ampicilina sódica ${ }^{\text {h }}\left(20 \mathrm{mg} \mathrm{kg}^{-1}\right), 30$ minutos antes do início da transfixação percutânea.

Para obtenção da biópsia, em cada cão foi identificado por palpação o músculo vasto-lateral e, próximo a sua inserção e sobre o seu ventre, a pele foi perfurada com uma agulha hipodérmica 16G. Em seguida, foi inserida no ventre muscular a agulha para biópsia do tipo "tru-cut" e removido um segmento muscular de aproximadamente $4,0 \times 2,0 \mathrm{~mm}$. O fragmento foi depositado em frasco estéril com formol para análise histomorfométrica. Este procedimento foi realizado nos dias zero, 30, 60 e 90 da imobilização.

Para imobilização do joelho, foi efetuada a fixação externa percutânea biplanar ou tipo II, conforme descrito por ARON (1996), mantendo a articulação numa angulação próxima a $90^{\circ}$, medida com o auxílio de um goniômetro. Como barra de conexão entre os pinos, foi utilizada resina acrílica autopolimerizável.

Os animais foram submetidos à terapia antiinflamatória com cetoprofeno $10 \%\left(2 \mathrm{mg} \mathrm{kg}^{-1}\right)$, por via subcutânea, durante três dias. A ferida de pele, feita durante a biópsia muscular, foi higienizada com solução salina isotônica e protegida por esparadrapo hipoalergênico. As áreas de inserções dos pinos, na pele, foram higienizadas com solução salina isotônica, protegidas por gaze embebida em solução de nitrofurazona e o aparelho de imobilização recoberto com atadura diariamente, durante 30 dias de imobilização.

Ciência Rural, v.37, n.1, jan-fev, 2007. 
Decorrido este período, os pinos que mantinham imobilizada a articulação do joelho foram removidos sob anestesia geral. Com o auxílio de um alicate específico, os pinos foram cortados em ambas as faces, aproximadamente $2,0 \mathrm{~cm}$ da pele, e posteriormente tracionados. Em seguida, foi realizada a higienização da área com solução salina isotônica e colocada atadura de crepe.

A EENM foi iniciada para os animais do grupo III após 48 horas da confecção do aparelho de imobilização rígida temporária do joelho, permanecendo por todo o período de avaliação, ou seja, durante (30 dias) e após a imobilização (60 dias) e, para os do grupo II, somente após a sua remoção (30 dias). A corrente emitida pelo aparelho de EENM tinha como característica onda pulsada unidirecional monofásica de baixa freqüência. Os parâmetros definidos no eletroestimulador foram freqüência de $50 \mathrm{~Hz}$, largura do pulso de 300 milissegundos, modo sincronizado, com ciclos de estimulação de 12 segundos seguidos por 25 segundos de descanso (relação ton:toff de 1:2). A rampa de subida (rise) e descida (decay) do pulso foi de três e cinco segundos, respectivamente. As sessões foram realizadas com o animal em decúbito lateral esquerdo, três vezes por semana, em dias alternados e com duração de acordo com o período preestabelecido dos grupos. A intensidade de corrente foi ajustada no limite de tolerância (vocalização, inquietude, retirada do membro estimulado) do animal ao estímulo. O tempo de estimulação em cada sessão de eletroterapia foi de 30 minutos.

Os músculos estimulados foram os que compõem o quadríceps femoral, ou seja, vasto lateral, vasto medial, vasto intermédio e reto femoral. Foi realizada a tricotomia da coxa direita e feita anti-sepsia com álcool para remoção da oleosidade e redução da resistência para passagem de corrente elétrica. Cada canal de corrente fica localizado no aparelho de EENM e é capaz de estimular um determinado músculo. Ele é composto por dois fios que são acoplados aos eletrodos de superfície. Nesta pesquisa, foram utilizados dois canais (Canais 1 e 2), sendo que um eletrodo de cada canal foi colocado sobre o ponto motor (THOMSON \& BOWEN, 1971) do músculo vasto medial (Canal 1face medial da coxa) e do vasto lateral (Canal 2 - face lateral da coxa). Os outros dois eletrodos restantes de ambos os canais permaneceram situados sobre os mesmos músculos, sendo que o eletrodo do canal 1 foi fixado na face lateral da coxa e sobre o músculo vasto lateral e o eletrodo do canal 2, na face medial e sobre o músculo vasto medial, promovendo o fechamento de corrente numa disposição diagonal, a fim de que a corrente propagasse também para os músculos reto femoral e vasto intermédio. Foi aplicado gel condutor apropriado de aproximadamente $3,0 \mathrm{~mm}$ de espessura sobre os eletrodos, posicionados conforme a técnica quadripolar (KITCHEN, 2003) e fixados com fita elástica.

Com a coxa tricotomizada em ambos os membros pélvicos, e com auxílio de uma fita maleável de escala métrica graduada em centímetros, foram marcados três pontos eqüidistantes entre o trocânter maior e o côndilo lateral do fêmur e realizada a mensuração da sua circunferência, para a obtenção de um valor médio. Com o auxílio de um goniômetro universal, foram mensuradas as angulações do joelho em flexão total e em extensão total para se obter a limitação do movimento. Esses procedimentos foram realizados no pré-imobilizado e aos 30,60 e 90 dias após imobilização.

Após a remoção do aparelho de fixação externa tipo II, os cães foram avaliados segundo a adaptação à marcha. A recuperação do uso funcional do membro foi estimada clinicamente, mediante cinco graus: I - o paciente não usa nem apóia o membro; II o paciente usa e apoia membro com pouca freqüência durante estação e ao caminhar, não sustentando o peso no membro afetado, elevando-o ao correr; III - uso claudicante do membro pelo paciente em estação e ao caminhar com sustentação parcial do membro, elevando-o ao correr; IV - o paciente caminha sem claudicar e posiciona normalmente o membro em estação, claudica ao correr sem elevá-lo; V - uso funcional do membro pelo paciente (TUDURY \& RAISER, 1985). Esta avaliação foi realizada pelo mesmo observador durante o pós-operatório, antes das sessões de EENM.

Foram coletados $8 \mathrm{ml}$ de sangue da veia jugular nos dias zero, 1, 7, 30, 45, 60, 75 e 90 de pósoperatório para obtenção do soro sangüíneo e avaliação da atividade das enzimas creatina-quinase (CK) e aspartato-amino-transferase (AST). Com exceção do dia zero, as coletas foram distribuídas em cinco tempos, ou seja, antes (T0), imediatamente após (T1), 6 (T2), 24 (T3) e 48 (T4) horas após o início da EENM.

Cada fragmento obtido na biópsia muscular foi identificado, fixado em formol, processado e corado pelo método de Hematoxilina e Eosina. Foram efetuados estudos morfométricos de mensurações de fibras musculares. Quando possível, foram realizadas 20 medidas de diferentes fibras ou diferentes locais nas fibras, respeitando-se uma distância mínima de $2 \mu$ entre cada medida.

Para análise estatística, foi utilizado o pacote estatístico SAS, versão 8.0. Os graus de claudicação foram avaliados pelo teste de Tukey, a goniometria e a circunferência da coxa pelos testes de GLM e por regressão quadrática, e a CK e a AST pelo teste de Tukey. Para morfometria, foram utilizados os testes de Tukey, de regressão linear e de regressão quadrática. 


\section{RESULTADOS E DISCUSSÃO}

O método de fixação percutânea tipo II, modificada conforme a técnica utilizada por KOSACHENCO et al. (1998), proporcionou rígida imobilização articular, oferecendo excelente estabilização, corroborando com os resultados de MAZZANTI et al. (2004), que imobilizaram a articulação do joelho após homoimplante ortotópico conservado na reparação tenopatelar em cães, e de ANDERSON \& CONSTANTINESCU (1998), que indicaram o uso do método para reparação de ligamento e de fraturas distais de membros. Neste experimento, no entanto, o emprego do método de imobilização rígida temporária com pinos de Steimann por 30 dias teve como objetivo determinar a atrofia por desuso principalmente dos músculos que compõem a coxa.

A freqüência utilizada de $50 \mathrm{~Hz}$ teve como base a informação de EVANGELISTA et al. (2003), que utilizaram a eletroestimulação, com freqüência modulada em $50 \mathrm{~Hz}$, para melhorar a performance no teste de aptidão física em militares, observando que estes foram beneficiados não só na estrutura muscular, mas também nas preparações físicas para o desporto e na redução do percentual de tecido adiposo. Outra justificativa para o emprego desta freqüência está no tipo de fibras musculares que predominam nos músculos estimulados neste experimento. ARMSTRONG et al. (1982) demonstraram que nos músculos que compõem o quadríceps femoral, com exceção do vasto intermédio, predominam as fibras tipo II, ou seja, de contração rápida, sendo citado por EVANGELISTA et al. (2003) que a freqüência ideal para contração destas fibras encontra-se entre 50 e $150 \mathrm{~Hz}$.

Os cães de todos os grupos atingiram o retorno funcional do membro (grau V) entre o $37^{\circ}$ (grupo

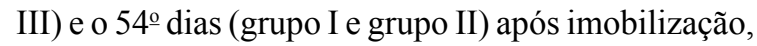
não havendo diferença significativa entre os grupos. A imobilização externa rígida temporária em flexão e a ausência de cirurgia articular provavelmente tiveram influência neste resultado, já que MILLIS (2004) afirmou que a imobilização em extensão resulta em maior espasticidade muscular e aumenta o tempo de recuperação funcional do membro, resultado este também encontrado por MAZZANTI (2002) e SOUZA \& MAZZANTI (2005), que imobilizaram o joelho em extensão após artroplastia.

Não houve diferença estatística entre os grupos na limitação do movimento articular. Logo após a remoção dos pinos de transfixação, ela se normalizou provavelmente pela ausência de cirurgia articular, sem a necessidade de empregar-se outras modalidades terapêuticas, como massageamento por deslizamento superficial, movimentação passiva da articulação, alongamento passivo e exercícios de caminhada descritos por SOUZA \& MAZZANTI (2005). Os valores da circunferência da coxa variaram no pósoperatório; no entanto, não apresentaram diferença estatisticamente significativa entre os grupos, demonstrando que esta mensuração é subjetiva, já que a EENM foi realizada em apenas quatro músculos e não em todos os que compõem a coxa. Logo, a mensuração da circunferência da coxa permitiu demonstrar o grau de atrofia dos músculos que formam a coxa e não o ganho de massa em grupos musculares estimulados isoladamente.

A biópsia do músculo vasto lateral foi de fundamental importância para a avaliação do efeito da EENM de baixa freqüência, já que os demais parâmetros não demonstraram diferença entre os grupos. A técnica de biópsia muscular com agulha "tru-cut" foi de fácil aplicação, podendo ser indicada para todos os programas de reabilitação em animais submetidos à eletroterapia com intuito de avaliar o ganho de massa muscular. Como limitação, pode-se citar a pequena quantidade de material às vezes retirada do músculo, $\mathrm{o}$ que impede a confecção de lâminas histológicas. Para evitar tal perda, recomendam-se duas a três colheitas de fragmentos musculares, além de considerar-se a curva de aprendizado, sendo que o treinamento prévio pode evitar essa complicação, a qual ocorreu, neste trabalho, na obtenção das primeiras biópsias. Não foram detectadas complicações decorrentes da biópsia, à semelhança de REYNOLDS et al. (1995), os quais concluíram que o procedimento foi livre de complicações graves, sendo uma alternativa confiável para a biópsia visando ao diagnóstico e ao manejo das miopatias.

Quanto à morfometria das fibras musculares longitudinais, verificou-se diferença altamente significativa $\mathrm{P}=0,0005$ entre os grupos. Os cães do grupo II obtiveram maior diâmetro das fibras musculares longitudinais, seguidos pelos cães do grupo III, em relação ao grupo controle (Figura 1). Este grupo foi o que apresentou a menor recuperação no tamanho das fibras musculares longitudinais. Pode-se verificar que o músculo vasto lateral dos cães do grupo controle, embora não sofresse EENM, teve um aumento no diâmetro das fibras em relação aos períodos preestabelecidos de pós-imobilização. Acredita-se que o retorno funcional do membro, juntamente com a caminhada, contribuíram para este resultado. Quanto aos cães dos grupos II e III, foi notado que os animais que receberam EENM somente após a remoção da imobilização rígida temporária apresentaram maior ganho de massa muscular, demonstrando que a EENM

Ciência Rural, v.37, n.1, jan-fev, 2007. 


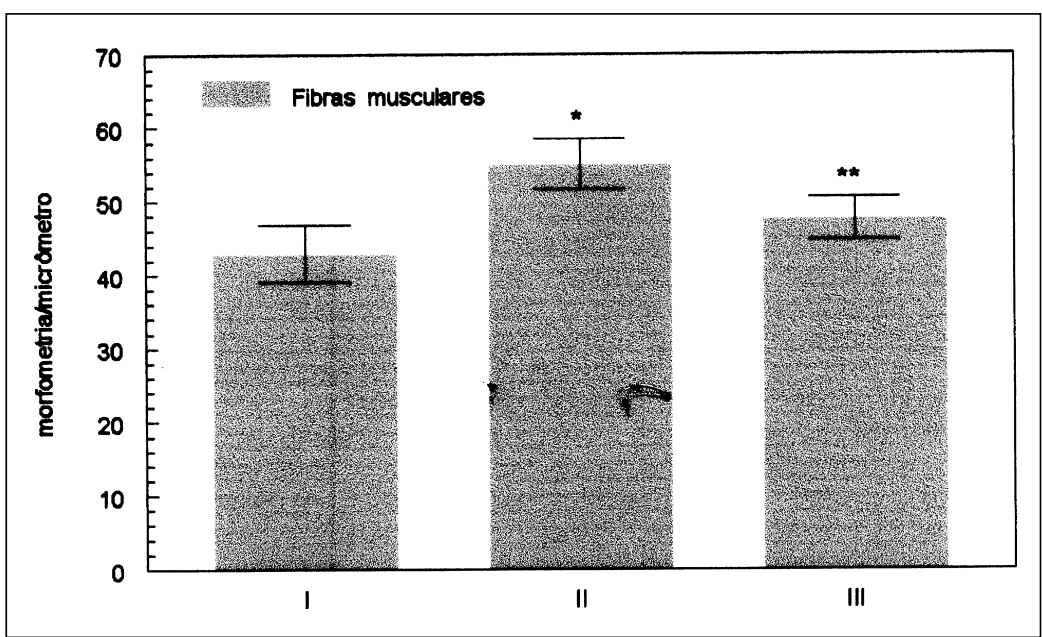

Figura 1 - Morfometria (micrômetro) das fibras musculares longitudinais aos 60 dias da imobilização rígida temporária do joelho. Grupo I ou controle, Grupo II: estimulação elétrica neuromuscular (EENM) após a retirada da imobilização rígida; e Grupo III: EENM durante e após a retirada da imobilização rígida do joelho. As barras representam a média \pm desvio padrão $\left({ }^{*} \mathrm{P}=0,0005, \mathrm{n}=4\right)$.

ideal (off time) deve ser de aproximadamente 60 segundos para evitar a fadiga muscular.

\section{CONCLUSÕES}

Diante dos resultados obtidos, pode-se concluir que o modelo utilizado induz a atrofia dos músculos da coxa e que a eletroestimulação neuromuscular de baixa freqüência ocasiona a hipertrofia do músculo vasto lateral somente após a remoção da imobilização, recomendandose o seu uso em cães submetidos à imobilização rígida temporária do joelho e que apresentem atrofia muscular.

\section{FONTES DE AQUISIÇÃO}

durante a imobilização (grupo III) não influenciou no aumento do diâmetro das fibras musculares. A presença do aparelho de fixação pode ter impedido o recrutamento de um maior número de fibras musculares, não sendo recomendada a EENM na presença de imobilização rígida articular.

O ciclo de estimulação de 1:2 (12 segundos “on" e 25 segundos “off”) utilizado é recomendado por JOHNSON \& LEVINE (2004) para evitar a fadiga muscular gerada pela contração muscular. Como métodos para avaliar a fadiga muscular, pode-se citar a mensuração das atividades enzimáticas, como a CK demonstrada por DAHLSTEDT et al. (2000), bem como os sinais clínicos de dor gerada pelos acúmulos de substâncias do metabolismo celular, conforme descrito por WESTERBLAD et al. (1998).

Os resultados das análises das enzimas $\mathrm{CK}$ e AST séricas não demonstraram diferença estatisticamente comprovada entre os três grupos. Os valores encontrados não foram suficientemente elevados para sugerir a ocorrência de lesões nas fibras musculares ou fadiga muscular, como citado por JOHNSON \& LEVINE (2004). Isso pode ser justificado pela escolha da relação "on time/off time" de 1:2, no tempo de estimulação utilizada no experimento, o que daria tempo para a recomposição da fosfogenase. Esta enzima foi citada por EVANGELISTA et al. (2003) como necessária para a contração muscular, sendo que, durante a EENM, pode ser consumida em 15 segundos e a duração para o seu reabastecimento imediato pode durar 22 segundos. Esse tempo é aproximado aquele citado por BRASILEIRO et al. (2002), em que o descanso
a-Pamoato de Pirantel/praziquantel - Petzi Plus/Vetbrands Jacareí-SP

b-Tiopental Sódico - Thiopentax

c-Sulfato de morfina - Dimorf/Cristália do Brasil - Itapira-SP

d-Maleato de acepomazina - Acepran 1\%/Univet - SP

e-Propofol - Diprivan/ICI Wellcome - SP

f-Halotano - Halotano/Hoechst Marion Roussel - SP

g-Cloridrato de Fentanila - Fentanest/Jansen-Ciliag Farmacêutica LTDA - SP

h-Ampicilina Sódica - Ampicillin/União Química - SP

i-Cetoprofeno 10\% - Ketofen/Merial - SP Nitrofurazona solução - IFAL, RS.

j-Neurodyn N-43 - Ibramed, Amparo, SP.

\section{COMITÊ DE ÉTICA E BIOSSEGURANÇA}

A utilização dos animais nesta pesquisa seguiu as normas do Colégio Brasileiro de Experimentação Animal e, ao término do experimento, foram castrados e doados.

\section{AGRADECIMENTO}

Ao Conselho Nacional de Desenvolvimento Científico e Tecnológico $(\mathrm{CNPq})$, pelo financiamento da pesquisa vinculada ao processo 550866/2002-7.

\section{REFERÊNCIAS}

ANDERSON, M.A.; CONSTANTINESCU, G. Using transarticular external skeletal fixaation devices. Veterinary Medicine, v.93, n.5, p.468-472, 1998.

ARMSTRONG, R.B. et al. Distribution of fiber types in locomotory muscles of dogs. American Journal Anatomy, v.163, n.1, p.87-98, 1982.

ARON, D.N. Tendões. In: BOJRAB, M.J. Técnicas atuais em cirurgia de pequenos animais. 3.ed. São Paulo: Roca, 
1996. Cap.40, p.516-527.

BRASILEIRO, J.S. et al. Parâmetros manipuláveis clinicamente na estimulação elétrica neuromuscular (EENM). Fisioterapia Brasil, v.3, n.1, p.16-24, 2002.

CARVALHO, C.M.M. Efeitos da imobilização e do exercício físico em algumas propriedades mecânicas do músculo esquelético. 2001. 61f. Dissertação (Mestrado em Interunidades em Bioengenharia) - EESC/FMRP/IQSC, Universidade de São Paulo.

DAHLSTEDT, A.J. et al. Is creatine kinase responsible for fatigue? Studies of isolated skeletal muscle deficient in creatine kinase. FASEB Journal, v.14, p.982-990, 2000.

EVANGELISTA, R.A. et al. Adaptação da característica fisiológica da fibra muscular por meio de eletroestimulação. Fisioterapia Brasil, v.4, n.5, p.326-334, 2003.

JOHNSON, J.; LEVINE, D. Electrical stimulation. In: MILLIS, D.L. et al. Canine rehabilitation \& physical therapy. Missouri: Elsevier, 2004. Cap.17, p.289-302.

KITCHEN, S. Eletroterapia. Prática baseada em evidência 11. ed. São Paulo: Manole, 2003. 348p.

KOSACHENCO, B.G; et al. Artrodese do joelho em cão pela fixação externa. Ciência Rural, v.28, n.2, p.271-275, 1998.

MAZZANTI, A. Homoimplante ortotópico conservado, associado à terapia "soft laser" na reparação tenopatelar em cão. 2002. 80f. Tese (Doutorado em Cirurgia) - Programa de Pós-graduação em Medicina, Veterinária da Universidade Federal de Santa Maria.

MAZZANTI, A. et al. Homoimplante ortotópico conservado, associado à terapia "soft laser" na reparação tenopatelar em cão. Ciência Rural, v.34, n.2, p.429-437, 2004.

MILLIS, D.L. Responses of musculoskeletal tissues to disuse and remobilization. In: MILLIS, D.L. et al. Canine rehabilitation \& physical therapy. Missouri: Elsevier, 2004. Cap.7, p.113-159.

MORRISSEY, M.C. et al. The effects of electrical stimulation on the quadriceps during postoperative knee immobilization. American Journal of Sports and Medicine, v.13, n.1, p.4045,1985

RAISER, A.G. Homoimplante ortotópico de tendão calcâneo comum, conservado em glicerina a $98 \%$, e tratado com radiação laser arseneto de gálio, sob dois métodos de imobilização, em cães. 2000. 88f. Tese (Doutorado em Cirurgia) - Programa de Pós-graduação em Medicina, Veterinária da Universidade Federal de Santa Maria.

REYNOLDS, A.J. et al. New approach to percutaneous muscle biopsy in dogs. American Journal Veterinary Research, v.56, n.8, p.982-985, 1995.

SOUZA, S.F.; MAZZANTI, A. Terapia física na análise da marcha em cães submetidos à artroplastia do joelho. Acesso em: 20dez.2005. On line. Disponível em: $<$ http:// www.ufsm.br/ppgmv/seminarios2005/soraiafdesouza.pdf.

THOMSON, F.K.; BOWEN, J.M. Eletrodiagnostic testing: mapping and clinical use of motor points in the dog. Journal American Veterinary Medical Association, v.159, n.12, p.1763-1771, 1971

TUDURY, E.A.; RAISER, A.G. Redução de fraturas distais de fêmur em cães, empregando pinos de Steinmann em substituição aos de Rush. Revista Centro de Ciências Rurais, v.15, n.2, p.141-155, 1985 .

WESTERBLAD, A.H. et al. Mechanisms underlying the reduction of isometric force skeletal muscle fatigue. Acta Physiol Scandinavia, v.162, n.1, p.253-260, 1998. 